\title{
Fiesta de la chonta y su impacto en el turismo comunitario del pueblo shuar
}

\section{Chonta party and its impact on community tourism of shuar people}

\author{
Vallejo Yucci, Timoteo Efraín ${ }^{1 *}$, León Fanacio, Priscilia Yuri ${ }^{1}$ y Torres Cueva , Dayana Nicolle ${ }^{1}$ \\ ${ }^{1}$ Universidad Católica de Cuenca, Sede Macas, Ecuador \\ tvallejo@ucacue.edu.ec
}

\begin{abstract}
Resumen
La Amazonía es una eminente fuente explotable turísticamente, donde su potencial y exuberante naturaleza con variedad de flora y fauna endémica presenta atractivos lugares de selva primaria como también sus ríos, lagos, cascadas, cavernas y a su pueblo de la etnia Shuar que habitan con costumbres ancestrales y entre ellos resaltamos la Fiesta de la chonta que celebran como parte de su cultura, es así que en la provincia de Morona Santiago existe estos eventos protagonizados por hombres y mujeres de esta etnia a la cual se desea dar el valor que un pueblo ancestral se merece con la Promoción de la Fiesta de la Chonta y sus ritos ancestrales como un producto atractivo en el Turismo Comunitario. Para alcanzar los objetivos de la investigación se aplicó una encuesta a la comunidad de San Luis de Ininkis tradicional pueblo Shuar en realizar este evento, como resultados valorativos de impacto local y nacional deja un espacio para que las entidades de desarrollo y gobiernos locales apoyen en la transformación del turismo comunitario.
\end{abstract}

Palabras clave: Chonta, Rituales, Turismo, Tradición, Cultura.

\begin{abstract}
The Amazon is an eminently exploitable tourist source, where its potential and exuberant nature with endemic flora and fauna presents attractive places of primary forest as well as its rivers, lakes, waterfalls, caverns and to its town of the ethnic group Shuar that inhabit with ancestral customs and among them we highlight the Chonta Festival celebrated as part of their culture, so in the province of Morona Santiago there are these events carried out by men and women of this ethnic group to which it is desired to give the value that an ancient people deserves with the Promotion of the Festival of the Chonta and its ancestral rites as an attractive product in the Community Tourism. In order to achieve the objectives of the research, a survey was carried out to the community of San Luis de Ininkis, traditional Shuar village, to carry out this event, as results of local and national impact assessments leave a space for development entities and local governments to support the transformation of community tourism.
\end{abstract}

Key words: Chonta, Rituals, Tourism, Tradition, Culture.

\section{Introducción}

El presente estudio tiene como principal objetivo determinar la fiesta de la chonta como atractivo turístico, para lo cual se requiere de información primaria y secundaria. Se conoce que el producto existe en la zona y que sirve para la alimentación, así como también se conoce que posee ciertas propiedades medicinales aún no comprobadas científicamente.

Debido a la importancia que tiene el producto por ser endémico y que su cosecha se realiza una sola vez al año, además por ser fuente de ritos ancestrales que mantienen las culturas en las comunidades Shuar, se ha tomado como objeto de estudio con la finalidad de incentivar en las futuras generaciones el valor ancestral de su cultura, valorar la producción nacional como fuente de riqueza e identidad cultural, incrementar la agricultura a través de programas sustentables dirigidos por gobiernos seccionales y mantener los ritos y tradiciones por medio de áreas académicas.

(Pereira Valarezo y Escobar, 2009) menciona en su libro que la Fiesta de la Chonta o rito Uwí, Es conocida como fiesta de la abundancia y está ligada a la cosmovisión del pueblo Shuar de la Amazonía ecuatoriana. Según los mayores, Uwí viene, todos los años, cargado de frutas pintonas, ya maduras. Uwí se lleva a los niños y a los ancianos. Para evitar que lo haga, se realiza una danza en su honor. Uwí es la chonta, una larga y espinosa palmera, y también es su fruto conocido como "chontaduro".

(Barrueco y Domingo, 2007) señala en su publicación que: "En las Comunidades se hace casa expresamente para celebrar la fiesta de la chonta. El hombre hace todo lo que 
es labor de la cosecha y recolección de alimentos, caza y pesca, para que la mujer sea quien prepare la chonta y la chicha y todos los alimento.

Es en el tiempo de la chonta cuando hacen la fiesta. Primero se comienza con la cosecha del fruto: el chontaruro; y sigue la preparación de los alimentos y bebida para convidar a los invitados a la fiesta. Ahí va maduro, yuca, elaboración de chicha; preparar en cantidad para todas las personas que iban a trabajar para hacer la casa, en que se hace la gran fiesta, porque debe haber comida en abundancia para todos".

La fiesta comienza en el momento de la recogida de la chonta. Se trata de un acto comunitario en el que participan pequeños grupos. Los hombres se dirigen a los lugares donde se encuentran las racimas maduras de chonta y las mujeres van detrás de ellos, portando el chankín -una especie de canasto tejido con fibras naturales que se sujeta en la cabeza desde la frente a la espalda o colgado al hombro.

En el proceso de preparación de la chicha, la pepa de chonta debe ser molida, lavada y cubierta con hojas para la cocción. El trabajo de las ancianas consiste en pelar las pepas y masticarlas, ensalivando la masa para su fermentación y moldeándola para colocarla en la olla, donde los demás ponen, al mismo tiempo, el fruto molido. Cuando la chicha está lista, por la noche, se congrega la comunidad en el lugar en el que se celebrará la danza, que dura aproximadamente seis horas y se ejecuta con los pies descalzos, a fin de entrar en contacto con la tierra.

La chonta es una palmera monopódica cilíndrica que alcanza de 12 a $15 \mathrm{~m}$ de altura, con tallo duro y liso de 12 a $15 \mathrm{~cm}$ de diámetro, de color cenizo oscuro, copa con $10 \mathrm{o}$ 12 hojas. Se la encuentra distribuida en toda la Amazonía. (Pereira Valarezo y Escobar, 2009)

Se espera proyectar la chonta como atractivo turístico, difundiéndolo al mercado local, nacional e internacional.

\section{Metodología}

Esta investigación se realizó en la Comunidad de San Luis de Ininkis pertenecientes a la Parroquia Sevilla Don Bosco, es a nivel descriptiva y Correlacional, usando encuestas y entrevistas para obtener la información.

\section{Tipo de investigación}

- Investigación Descriptiva: El propósito de esta investigación es describir cómo se manifiestan determinados fenómenos. En la realización de este estudio se utilizará la modalidad bibliográfica documental puesto que su desarrollo se basará en información existente en libros, tesis, artículos y demás documentos pertinentes.

- Investigación Correlacional: Este tipo de investigación tiene como fin medir el grado de incidencia entre las variables que atañen el problema.

\section{Población y Muestra}

Según la población utilizada para el cálculo de la muestra (tabla 1), estadísticamente se tiene que en la Comunidad de San Luis de Ininkis, existe una población de
900 habitantes mayores de 18 años, registrada anualmente por el Síndico de la Comunidad.

Tabla 1

Habitantes Comunidad San Luis de Ininkis

\begin{tabular}{|l|r|}
\hline \multicolumn{2}{|c|}{ Comunidad de San Luis de Ininkis } \\
\hline Habitantes 2016 & Numero \\
\hline Personas Mayores de 18 Años & 900 \\
\hline
\end{tabular}

Fuente: Sindico de la Comunidad de San Luis de Ininkis. Elaborado: Autores

El presente estudio de investigación pertenece a un método de investigación descriptiva, con estudios de campo, utilizando en primer lugar la técnica de la encuesta mediante la muestra de una población finita que correspondió a 88 familias, las cuales fueron aplicadas con la siguiente formula:

Para el cálculo de la muestra a utilizarse se determina un nivel de confianza del $95 \%$ y un margen de error del $5 \%$. Para obtener la muestra de una población finita se aplicó la siguiente fórmula:

$$
n=\frac{N \times Z_{a}^{2} \times p \times q}{d^{2} \times(N-1)+Z_{a}^{2} \times p \times q}
$$

Cálculo de la muestra: La metodología que se aplicó para la investigación será el método cualitativo y cuantitativo el cual nos llevó a tener una investigación aplicada por lo que fue una investigación más práctica.

$$
\begin{aligned}
& \mathrm{N}=\text { Tamaño de la población } \\
& \mathrm{d} 2=\text { Error de estimación } \\
& \mathrm{d} 2=5 \%=(0.05) 2 \\
& \mathrm{Z} 2=\text { Grado de Confianza } \\
& Z 2=95 \%=(1,96) 2 \\
& P . Q=\text { valores constantes; } \mathrm{P} . \mathrm{Q}=50 \%=(0,5)(0,5)
\end{aligned}
$$

Para la entrevista se utilizó el método Sistémico, con la técnica de la Entrevista e Instrumento la Guía de Entrevista que se aplicó al Especialista de Gestión y Promoción del Ministerio de Turismo, Ing. Cristian Jara Suarez, la cual consistió en cuatro preguntas.

\section{Resultados}

FACTOR N ${ }^{\mathbf{0}} 1$ : Fiesta de la chonta en la actualidad

La totalidad de la población Shuar encuestada confirma que actualmente practican todos los rituales que contiene la fiesta de la chonta, sin dejar espacio a la duda se pudo constatar que ellos obedecen y respetan una costumbre ancestral y difícilmente lo dejaran de hacer a través del tiempo Gráfico 1.

\section{FACTOR N 2: Actividades relevantes de la fiesta} de la chonta

Se pudo constatar que la actividad relevante en la tradicional fiesta de la chonta es para ellos la preparación de la bebida o brebaje conocido como "Chicha de chonta". Este proceso tiene una duración de 12 a 14 horas iniciando 


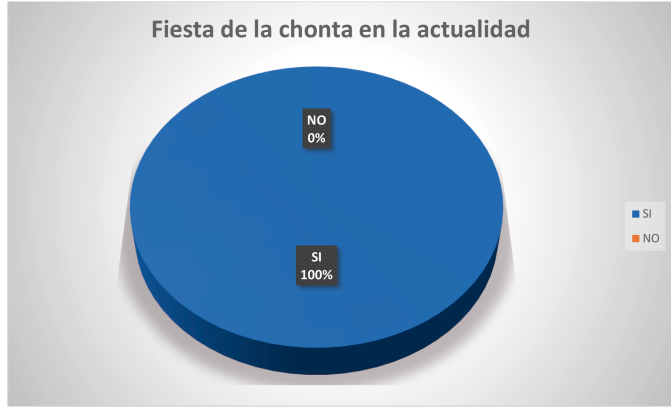

Figura 1. Fiesta de la chonta en la actualidad

Fuente: Encuestas realizadas en la Comunidad de San Luis Ininkis, 2017 Autor: Dayana Torres, Priscila León

con la cosecha del fruto e interactuando con deportes, danzas, y rituales aproximadamente hasta las doce de la noche extendiéndose la celebración hasta altas horas de la madrugada. (Gráfico 2)

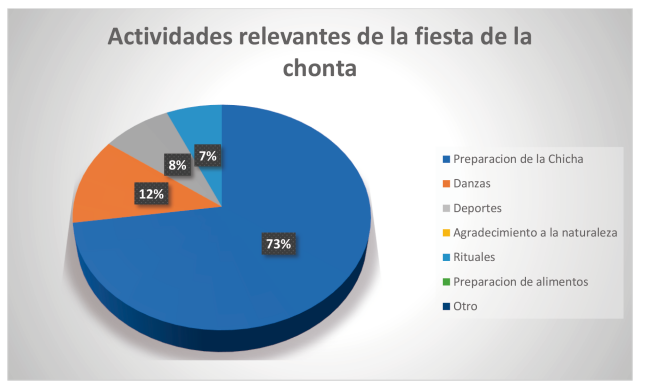

Figura 2. Actividades relevantes de la fiesta de la chonta

Fuente: Encuestas realizadas en la Comunidad de San Luis Ininkis, 2017 Autor: Dayana Torres, Priscila León chonta

FACTOR No3: Motivo de realizar la fiesta de la

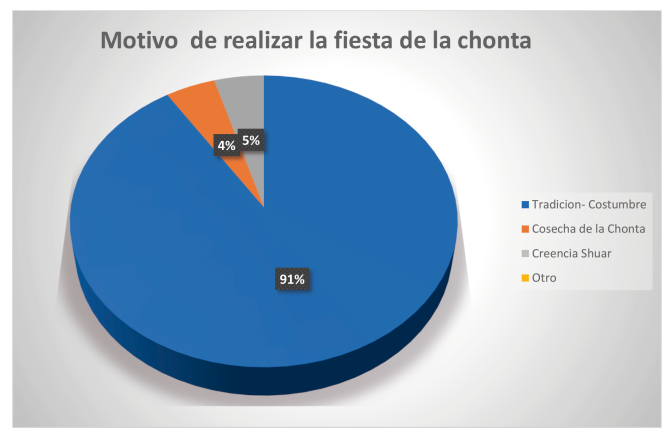

Figura 3. Motivo de realizar la fiesta de la chonta

Fuente: Encuestas realizadas en la Comunidad de San Luis Ininkis, 2017 Autor: Dayana Torres, Priscila León

La razón principal por la que este pueblo milenario de etnia Shuar, practican la fiesta de la chonta manifiesta que es por una típica tradición y costumbre cultural por lo que lo hacen dos veces al año por la producción natural del fruto conocido como chonta que inicia desde febrero hasta mayo de cada año. La mínima parte indica que el motivo de realizar la festividad es por la cosecha del fruto. Este es un indicar que el ministerio de turismo en la entrevista confirma que conocen como y cuando la etnia Shuar celebra 1 cosecha de la chonta con rituales de tradición propia de su cultura. (Gráfico 3)

FACTOR No4: Fiesta de la chonta como actividad para el turismo comunitario

El pueblo Shuar mantiene una expectativa económica, proponiendo este fiesta tradicional como evento de turismo comunitario nacional e internacional, comprometiendo a cooperar con una institución pública o privada que apoye sus costumbres, tradiciones, rituales y el entorno natural atractivo propios de la selva. (Gráfico 4)

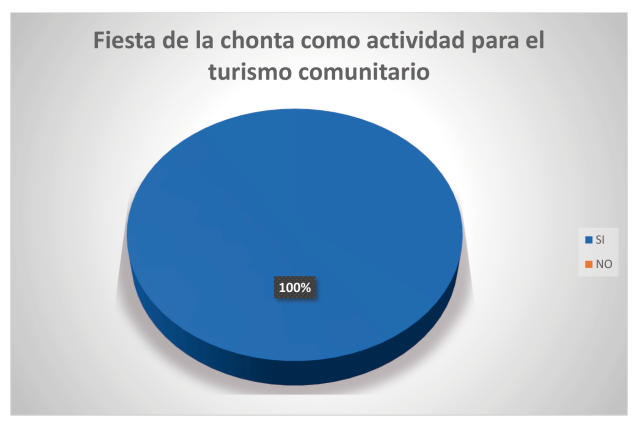

Figura 4. Fiesta de la chonta como actividad para el turismo comunitario

Fuente: Encuestas realizadas en la Comunidad de San Luis Ininkis, 2017 Autor: Dayana Torres, Priscila León

\section{FACTOR N5: Ámbito de aplicación para la Fiesta de la Chonta}

Existe la decisión unánime del pueblo Shuar en difundir esta tradicional fiesta de la chonta desde el micro entorno comunitario hacia el mercado nacional e internacional como un proyecto turístico de corto a mediano plazo. Corroborando esta pregunta de la encuesta con la segunda de la entrevista realizada al funcionario responsable del ministerio de turismo, se pudo entender que la institución está dispuesta a trabajar en forma coordinada con el pueblo Shuar para potencializar y difundir como un evento de proyecto turístico cultural primero a los turistas locales, y luego dispersar al turismo nacional e internacional, puntualizando que es necesario afianzar convenios con responsabilidad compartida para que no resulte una actividad paternalista por parte de los gobiernos locales, ya que durante los últimos años el costo económico y social ha repercutido como un gasto solo para los municipios. (Gráfico 5)

FACTOR Nº: Vinculación hacia el Ministerio de Turismo

El pueblo Shuar asentado en la comunidad San Luis de Ininkis perteneciente a la parroquia Sevilla Don Bosco, manifiesta su disponibilidad para trabajar con el ministerio de turismo y conseguir fidelizar una calificación para que la 


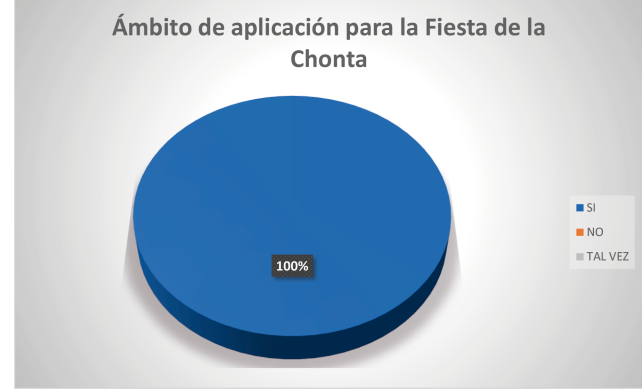

Figura 5. Ámbito de aplicación para la Fiesta de la Chonta

Fuente: Encuestas realizadas en la Comunidad de San Luis Ininkis, 2017 Autor: Dayana Torres, Priscila León

fiesta de la chonta ya forme parte de un atractivo turístico cultural que propicie el Ecuador.

Ante este punto de vista el ministerio de turismo contrasta su preocupación y responsabilidad de vincular en los futuros proyectos turísticos al pueblo Shuar dentro del sector cultural de la zona 6 a la que pertenece la provincia de Morona Santiago, reconociendo que si existe la voluntad de los involucrados pondrán en marcha este tipo de evento cultural como tradición y simbolismo de un sector rural del Ecuador.(Gráfico 6)

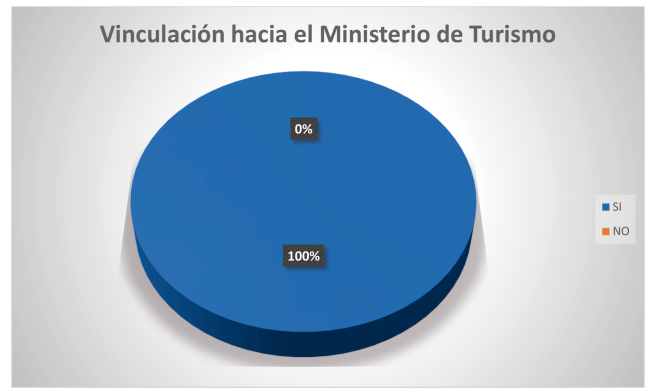

Figura 6. Vinculación hacia el Ministerio de Turismo

Fuente: Encuestas realizadas en la Comunidad de San Luis Ininkis, 2017 Autor: Dayana Torres, Priscila León

\section{FACTOR No7:Vinculación hacia el Ministerio de}

\section{Turismo}

Con la celebración de la fiesta de la chonta el pueblo Shuar se siente orgulloso de la cultura tradicional heredada de sus ancestros y manifiestan que tiene un impacto muy atractivo en sus costumbres sociales y los rituales como una forma de agradecer a su naturaleza. Con este antecedente que el pueblo Shuar califica de atractiva su tradicional Fiesta de la Chonta, el Ministerio de Turismo solo está para gestionar, fomentar y motivar los eventos culturales manifestando que el GAD municipal por medio de la COOTAD tiene la competencia de aprobar partidas presupuestarias que cubran la logística del evento cultural conocido y no apreciado por los otros sectores sociales. Entonces los GAD municipales existentes en la amazonia ecuatoriana tienen la responsabilidad financiera, moral y cultural de coadyuvar y

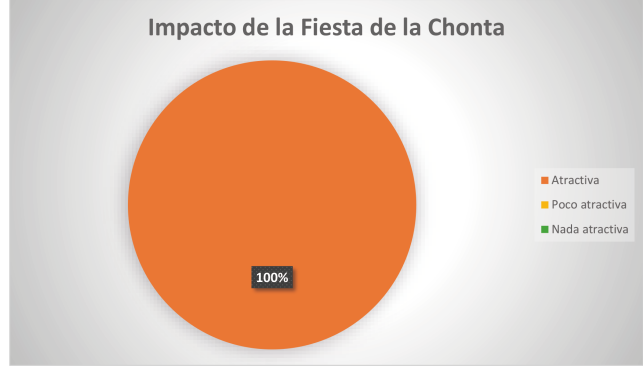

Figura 7. Vinculación hacia el Ministerio de Turismo

Fuente: Encuestas realizadas en la Comunidad de San Luis Ininkis, 2017 Autor: Dayana Torres, Priscila León

fortalecer las buenas costumbres y valores que posee cada región. (Gráfico 7)

\section{Conclusiones}

El pueblo Shuar de la amazonia ecuatoriana nunca dejara esta tradición de realizar la Fiesta de la Chonta entre una o dos veces al año, ya que recuerdan a sus ancestros, por la tradición y costumbre, realizan rituales, danzas, elaborando una bebida de la fruta de la chonta conocida vulgarmente como "chicha" para festejar la cosecha, agradeciendo de esta manera a la naturaleza.

Todo este proceso se realiza al interior de las comunidades de los pueblos Shuar pero todavía no está calificado este evento como un atractivo turístico por falta de apoyo y coordinación entre las entidades que intervengan en el desarrollo cultural de la región.

El Ministerio de Turismo, los diferentes gobiernos locales y el pueblo Shuar en cuanto al turismo comunitario no tienen convenios programados en especial para la fiesta de la chonta y formen parte de los eventos culturales en la amazonia ecuatoriana.

El pueblo Shuar califica de atractivo su fiesta y rituales de la chonta, pero en la actualidad no es valorado social y culturalmente como un evento que puede generar réditos económicos como turismo comunitario, ya que falta promocionar local, nacional e internacional.

\section{Referencias Bibliográficas}

Alberto, C., y Andrade, C. (1985). Danzas y bailes en el ecuador. , 6(2), 166. Descargado de http://www.jstor.org/stable/ 780200 ?origin=crossref doi: 10.2307/ 780200

Barrueco, S. D. B., y Domingo, P. (2007). La fiesta del uwí: Crónicas, tradiciones y entrevistas. Descargado de http: / / dspace.ups.edu.ec/ bitstream/123456789/11720/1/ mito\%20ultima20del\%20uwi.pdf

Broseghini, S., y Juank, A. (1978). La iglesia shuar : nueva presencia y nuevo lenguaje. Centro de Documentación, Investigación y Publicaciones,. 
Descargado de http: / / dspace.ups.edu.ec/ handle/123456789/11505

Pereira Valarezo, J., y Escobar, T. (2009). La fiesta popular tradicional del ecuador (1. ed ed.) (n. ${ }^{\circ}$ ). Fondo Editorial Ministerio de Cultura. Descargado de http://www.flacsoandes.edu.ec/ libros/digital/52864.pdf

Recibido: 10 de julio de 2017

Aceptado: 5 de septiembre de 2017 
